\title{
The Surface of Titan from Adaptive Optics Observations
}

Seran G. Gibbard, Bruce A. Macintosh, Claire E. Max

Lawrence Livermore National Laboratory, Livermore, CA 94550, USA

Henry Roe, Imke de Pater

University of California, Berkeley, CA 94720-3411, USA

E. F. Young

Southwest Research Institute, San Antonio, TX 78228-0510, USA

Christopher P. McKay

NASA Ames Research Center, Moffett Field, CA 94035, USA

\begin{abstract}
Saturn's largest moon Titan is the only satellite in the Solar System with a substantial atmosphere. Photolysis of methane creates a hydrocarbon haze in Titan's atmosphere that is opaque to visible light. The new adaptive optics system on the 10-meter W. M. Keck Telescope enables us to observe Titan with a resolution of 0.04 arcseconds, or 20 resolution elements across the disk. By observing at near-infrared wavelengths that are methane band windows we can see through Titan's hydrocarbon haze to the surface beneath. Recent adaptive optics images of Titan both in broadband ( $\mathrm{J}, \mathrm{H}$, and $\mathrm{K})$ filters and in narrowband filters that selectively probe Titan's surface and atmosphere allow us to determine surface albedo and properties of the hydrocarbon haze layer. Future observations will include high-resolution spectroscopy coupled with adaptive optics to obtain spectra of individual surface features.
\end{abstract}

